\title{
Dissociated Pmch and Cre Expression in Lactating Pmch-Cre BAC Transgenic Mice
}

\author{
Bethany G. Beekly 1,2, William C. Frankel'1,3, Tova Berg ${ }^{1}$, Susan J. Allen', \\ David Garcia-Galiano1, Giancarlo Vanini ${ }^{2,4}$ and Carol F. Elias 1,2,5*
}

\begin{abstract}
${ }^{1}$ Department of Molecular and Integrative Physiology, University of Michigan, Ann Arbor, MI, United States, ${ }^{2}$ Neuroscience Graduate Program, University of Michigan, Ann Arbor, MI, United States, ${ }^{3}$ Baylor College of Medicine, Houston, TX, United States, ${ }^{4}$ Department of Anesthesiology, School of Medicine, University of Michigan, Ann Arbor, MI, United States,
\end{abstract} ${ }^{5}$ Department of Obstetrics and Gynecology, School of Medicine, University of Michigan, Ann Arbor, MI, United States

\section{OPEN ACCESS}

Edited by:

Laurent Gautron,

University of Texas Southwestern Medical Center, United States

Reviewed by: Melissa Chee, Carleton University, Canada Qingchun Tong, University of Texas Health Science Center at Houston, United States

*Correspondence: Carol F. Elias cfelias@umich.edu

Received: 08 July 2020 Accepted: 04 August 2020 Published: 21 August 2020

Citation: Beekly BG, Frankel WC, Berg T, Allen SJ, Garcia-Galiano D, Vanini G and Elias CF (2020) Dissociated Pmch and Cre Expression in Lactating

Pmch-Cre BAC Transgenic Mice. Front. Neuroanat. 14:60. doi: 10.3389/fnana.2020.00060
The melanin-concentrating hormone $(\mathrm{MCH})$ system plays a role in many physiological processes including reproduction and lactation. However, research regarding the function of $\mathrm{MCH}$ on different aspects of the reproductive function lags, due in part to a lack of validated genetic models with which to interrogate the system. This is particularly true in the case of female reproduction, as the anatomy and function of the $\mathrm{MCH}$ system is not well-characterized in the female mouse. We set out to determine whether the commercially available Pmch-Cre transgenic mouse line is a viable model to study the role of $\mathrm{MCH}$ neurons in distinct female reproductive states. We found that Pmch is transiently expressed in several nuclei of the rostral forebrain at the end of lactation. This includes the medial subdivision of the medial preoptic nucleus, the paraventricular nucleus of the hypothalamus, the ventral subdivision of the lateral septum, the anterodorsal preoptic nucleus and the anterodorsal nucleus of the thalamus. The Pmch expression in these sites, however, does not reliably induce Cre expression in the Pmch-Cre (BAC) transgenic mouse, making this line an inadequate model with which to study the role of $\mathrm{MCH}$ in behavioral and/or neuroendocrine adaptations of lactation. We also contribute to the general knowledge of the anatomy of the murine $\mathrm{MCH}$ system by showing that lactation-induced Pmch expression in the rostral forebrain is mostly observed in GABAergic (VGAT) neurons, in contrast to the typical $\mathrm{MCH}$ neurons of the tuberal and posterior hypothalamus which are glutamatergic (VGLUT2).

Keywords: preoptic area, VGAT, VGLUT2, hypothalamus, sex-differences

\section{INTRODUCTION}

The melanin-concentrating hormone $(\mathrm{MCH})$ system has been implicated in a diverse array of fundamental physiological processes such as metabolic regulation, stress response, and sleep (Baker et al., 1985; Bahjaoui-Bouhaddi et al., 1994; Qu et al., 1996; Verret et al., 2003). Several studies have provided compelling evidence that $\mathrm{MCH}$ neurons also regulate aspects of reproductive function such as the release of luteinizing hormone (LH) and lactation (Knollema et al., 1992; Attademo et al., 2006). 
While MCH neurons have widespread projections throughout the brain, they originate from a discrete region of the hypothalamus (Bittencourt et al., 1992; Elias and Bittencourt, 1997; Bittencourt and Elias, 1998; Elias et al., 1998; Casatti et al., 2002; Sita et al., 2007). In rats, MCH expression is almost exclusively restricted to cell bodies in the incertohypothalamic area (IHy, aka rostromedial zona incerta or ZIm), the lateral hypothalamic area (LHA), and the perifornical area (PFx). The distribution of $\mathrm{MCH}$ neurons in mice and rats - and, indeed, in all rodents in which the system has been studied - follows a similar basic plan. However, close study of the mouse and rat reveals some key differences, including a population of periventricular $\mathrm{MCH}$ neurons seen in rats but not in mice, and the absence of more posterior groups in the mouse (Bittencourt et al., 1992; Diniz et al., 2019). Interestingly, $\mathrm{MCH}$ is transiently observed in the medial preoptic area (MPO), the periventricular preoptic nucleus, and the most rostral parts of the paraventricular nucleus of the hypothalamus (PVH) of female rats during lactation (Knollema et al., 1992; Rondini et al., 2010) and, to a lesser extent, in the MPO of the mouse (Knollema et al., 1992; Rondini et al., 2010; Diniz et al., 2019). The role of MPO MCH expression in the lactating rodents is unclear, potentially due to a lack of validated models with which to study this phenomenon. Indeed, functional studies in mice lag behind the anatomy of the $\mathrm{MCH}$ system, as the majority of the experiments referenced up to this point which involve reproductive function were performed in rats.

In the present study, we sought to determine whether the commercially available $\mathrm{Tg}(P m c h$-cre $) 1$ Lowl/J ("Pmch-Cre") transgenic mouse line (Kong et al., 2010) is a viable model to interrogate the role of the $\mathrm{MCH}$ system in female physiology. The Pmch-Cre mouse was developed using a bacterial artificial chromosome (BAC) containing the coding region for Cre recombinase flanked by upstream and downstream regulatory elements of the Pmch gene (Kong et al., 2010). This technique is moderately efficient and used to be a common method of introducing DNA sequences in the genome of a model organism. However, the loci of insertion for the BAC is random: it is incorporated into a genomic site with a unique set of regulatory elements, potentially imposing additional layers of regulation on Cre expression. It may also fail to recapitulate epigenetic regulation that occurs at the native gene locus and relies on enhancers that may be located hundreds of thousands of bases up- or downstream (Dean, 2006; Krivega and Dean, 2012). The unpredictable nature of this method necessitates thorough validation, which has not been performed in female mice, particularly in lactating dams.

In addition to defining the locations of $\mathrm{MCH}$ expression during lactation, we sought to determine whether these $\mathrm{MCH}$ neurons potentially release $\gamma$-aminobutyric acid (GABA), glutamate, both, or neither of the classical fast neurotransmitters. We used brain tissue from Cre-driven reporter lines expressing GFP in neurons expressing the vesicular glutamate transporter (VGLUT2) or the vesicular GABA transporter (VGAT) to verify the fast neurotransmitter phenotype of $\mathrm{MCH}$ neurons in different brain regions.

\section{MATERIALS AND METHODS}

\section{Mice}

\section{Animal Care}

Mice were housed in a vivarium at the University of Michigan with a 12/12 light/dark cycle and ad libitum access to food and water. The mice received phytoestrogen-reduced Envigo diet 2016 (16\% protein/4\% fat), except during breeding when mice were fed phytoestrogen-reduced Envigo diet 2019 (19\% protein/8\% fat). Phytoestrogen-reduced diet is routinely used in our laboratory to avoid the effects of exogenous estrogens on mouse physiology. All procedures and experiments were carried out in accordance with the guidelines established by the National Institutes of Health Guide for the Care and Use of Laboratory Animals and approved by the University of Michigan Committee on Use and Care of Animals (Animal Protocol \#8712).

\section{Pmch-Cre;eGFP Mice}

Commercially available Pmch-Cre $\left[\mathrm{Tg}(P m c h\right.$-Cre $) 1$ Lowl/J, JAX ${ }^{\circledR}$; stock \#014099] mice were used. This mouse model was developed using a BAC containing a Cre coding sequence downstream of the mouse pro-melanin concentrating hormone (Pmch) promoter (Kong et al., 2010).

The Pmch-Cre mice were crossed with two different mouse lines carrying Cre-inducible reporter genes. First, Pmch-Cre mice

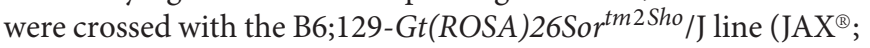
stock \#004077, “R26-eGFP”). This mouse carries a targeted mutation of the R26 locus with a loxP-flanked transcriptional blocking cassette preventing the expression of CAG promoterdriven enhanced green fluorescent protein (eGFP) reporter (Mao et al., 2001). Cre-mediated excision of the blocking cassette in Pmch-Cre;R26-eGFP mice results in expression of GFP in cells expressing Cre.

Pmch-Cre mice were also crossed with the B6;129S4Gt(ROSA)26Sor ${ }^{t m} 9(E G F P / R p l 10 a) A m c / J$ line (JAX ${ }^{\circledR}$; stock \#024750, “eGFP-L10a”), kindly donated by Dr. David Olson (University of Michigan), to generate Pmch-Cre;eGFP-L10a reporter mice. The eGFP-L10a mice express a chimeric L10a ribosomal subunit fused to eGFP. Successful Cre-mediated excision results in expression of the eGFP:L10A fusion protein in Cre-expressing tissues (Liu et al., 2014). Due to the ribosomal eGFP fusion, this mouse model enables a strong fluorescent signal to accumulate in cell bodies.

\section{Vgat-Cre;eGFP and Vglut2-Cre;eGFP Mice}

Vgat-IRES-Cre:Slc32a1 ${ }^{\text {tm } 2(\mathrm{cre}) \text { Lowl /J }}\left(\right.$ JAX $^{\circledR}$ mice, Stock \#016962) and Vglut2-IRES-Cre: Slc17a6 $6^{\text {tm } 2(\mathrm{cre}) \text { Lowl/J }}$ (JAX ${ }^{\circledR}$ mice, Stock \#016963) mice were crossed with eGFP-L10a reporter to generate mice which express GFP in VGAT- or VGLUT2-expressing cells, respectively (Vgat-Cre;eGFP-L10a and Vglut2-Cre;eGFP-L10a mice) (Vong et al., 2011).

\section{Genotyping}

All mice were genotyped before experiments by extracting DNA from tail tip biopsies using the REDExtract-N-Amp ${ }^{\text {TM }}$ Tissue PCR Kit, catalog no. XNAT (Sigma-Aldrich ${ }^{\circledR}$ ). PCR was performed with a Bio-Rad $\mathrm{C} 1000^{\mathrm{TM}}$ Thermal Cycler and included positive (DNA from the original JAX mice) and 
negative (sterile water) controls. Primer sequences can be found in Table 1.

\section{Experimental Groups}

Sexually naïve adult male Pmch-Cre;eGFP mice (8-12 weeks of age) were used. Adult female mice were divided into sexually naïve (8-10 weeks of age) and lactating groups (16-18 weeks of age) for a total of three experimental groups ( $n=4$ per group). An additional group of females $(n=4)$ was evaluated 5 days after weaning of the offspring as control for the lactation group. Experimental groups were evaluated in both reporter lines: PmchCre;R26-eGFP and Pmch-Cre;eGFP-L10a.

Additionally, Vgat-Cre;eGFP-L10a and Vglut2-Cre;eGFPL10a dams were bred with Vglut2-Cre and Vgat-Cre males to generate two more lactation groups in which to assess the fast neurotransmitter phenotype of $\mathrm{MCH}$ neurons ( $n=3$ per group).

\section{Perfusion and Histology}

Adult male Pmch-Cre;eGFP mice were euthanized and brains harvested between 8 and 12 weeks of age. Sexually naïve females, 8-10 weeks of age, were ascertained to be in diestrus before tissue was harvested. Brains were collected from lactating females on day 19 of lactation (16-18 weeks of age).

All mice were anesthetized with isoflurane and transcardially perfused with saline prepared with diethyl pyrocarbonate (DEPC)-treated water followed by $10 \%$ neutral buffered formalin for $10 \mathrm{~min}$. Following perfusion, brains were dissected and postfixed in $20 \%$ sucrose $/ 10 \%$ buffered formalin overnight at $4^{\circ} \mathrm{C}$, then cryoprotected in $20 \%$ sucrose in DEPC-treated $1 \times$ phosphate buffered saline (PBS) overnight at $4^{\circ} \mathrm{C}$. Brains were sectioned with a freezing Leica SM2010 R microtome (4 series, 30- $\mu \mathrm{m}$ thickness, in the frontal plane). Sections were stored at $-20^{\circ} \mathrm{C}$ in RNAse-free cryoprotectant (20\% glycerol, 30\% ethylene glycol in DEPC-PBS).

TABLE 1 | Primer sequences used for genotyping mice and performing radioactive in situ hybridization.

\begin{tabular}{ll}
\hline Primer & Sequence \\
\hline $\begin{array}{l}\text { Pmch-Cre (genotyping) } \\
\text { Common }\end{array}$ & GAA AAG ATA AGG CCT TCA AGT GCT \\
Internal Positive Control & GAT CT TCT GCA GTA TCT TCC TTC \\
Reverse & \\
Transgene Reverse & ATC GAC CGG TAA TGC AGG CAA \\
R26-eGFP (genotyping) & \\
Forward & AAGTTCATCTGCACCACCG \\
Reverse & TCCTTGAAGAAGATGGTGCG \\
L10-eGFP (genotyping) & \\
Forward 1 & GAG GGG AGT GTT GCA ATA ACC \\
Forward 2 & TCT ACA AAT GTG GTA GAT CCA GGC \\
Reverse & CAG ATG ACT ACC TAT CCT CCC \\
Pmch cDNA Template (ISH) & \\
Forward & (CAG AGA TGC AAT TAA CCC TCA CTA AAG \\
& GGA GA) AGC ATC AAA CTA AGG ATG GCA \\
Reverse & (CCA AGC CCT CTA ATA CGA CTC ACT ATA \\
& GGG AGA) GCA TAC ACC TGA GCA TGT CAA AA
\end{tabular}

\section{Radioactive in situ Hybridization}

Single-label radioactive in situ hybridization (ISH) was performed on one series of brain sections from each animal. Sections were mounted onto RNAse-free SuperFrost Plus slides (Fisher Scientific). Mounted tissue was fixed in 4\% paraformaldehyde in DEPC-treated $1 \times$ PBS for $20 \mathrm{~min}$, dehydrated in increasing concentrations of ethanol, cleared of lipids using xylenes, and subsequently rehydrated in decreasing concentrations of ethanol. The slides were pretreated by microwaving in sodium citrate buffer $(\mathrm{pH}$ 6.0), then dehydrated again in increasing concentrations of ethanol, air-dried, and stored at $-20^{\circ} \mathrm{C}$ (Sibony et al., 1995; Frazão et al., 2013; Mohsen et al., 2017).

The Pmch cDNA (template) was produced from mouse hypothalamic RNA and used to amplify a 478 bp sequence in the coding region of the Pmch gene (IDT, Inc.). Primer sequences are delineated in Table 1. The Pmch riboprobe was generated by in vitro transcription using ${ }^{35} \mathrm{~S}$-UTP as the radioisotope. ${ }^{35} \mathrm{~S}$ labeled Pmch probe was diluted in hybridization solution (50\% formamide, $10 \mathrm{mM}$ Tris- $\mathrm{HCl} \mathrm{pH} 8.0,0.01 \%$ of yeast tRNA, $0.05 \%$ of total yeast RNA, $10 \mathrm{mM}$ dithiothreitol/DTT, $10 \%$ dextran sulfate, $0.3 \mathrm{M} \mathrm{NaCl}, 1 \mathrm{mM}$ EDTA, and $1 \times$ Denhardt's solution) and applied to slides, which were allowed to hybridize overnight at $55^{\circ} \mathrm{C}$, as routinely done by our group (Frazão et al., 2013). Following post-hybridization treatment with RNAse, stringency washes with saline sodium citrate (SSC) buffer and dehydration in increasing concentrations of ethanol, sections were dried at room temperature and slides were placed in X-ray film cassettes with BMR-2 film (Kodak, Rochester, NY, United States) for 1 day (tuberal and posterior levels of the hypothalamus) or 4 days (rostral forebrain). Slides were then dipped in NTB autoradiographic emulsion (Kodak), dried for $3 \mathrm{~h}$ and stored in light-protected slide boxes at $4^{\circ} \mathrm{C}$ for 6 days (tuberal and posterior levels of the hypothalamus) or 14 days (rostral forebrain). Slides were developed with Dektel developer (Kodak, VWR, Radnor, PA, United States), dehydrated in increasing concentrations of ethanol, cleared in xylenes and coverslipped with DPX mounting medium (Electron Microscopy Sciences).

\section{Dual-Label Immunohistochemistry}

Dual-label immunohistochemistry (IHC) for $\mathrm{MCH}$ or NEI and GFP was performed in one series of brain sections from each animal. Sections were blocked for $30 \mathrm{~min}$ in 3\% normal donkey serum (NDS) and incubated overnight in chicken anti-GFP (1:10,000, Aves Labs, AB_2307317) and either rabbit anti-MCH (1:5,000, Phoenix Pharmaceuticals, AB_2722682) or rabbit antiNEI $(1: 15,000$, kindly provided by Dr. Paul Sawchenko, Salk Institute for Biological Studies, La Jolla, CA, United States) followed by secondary antisera (goat anti-chicken conjugated to Alexa Fluor ${ }^{\mathrm{TM}} 488$ AB_2534096, and donkey anti-rabbit conjugated to Alexa Fluor ${ }^{\mathrm{TM}} 594$ AB_141637) for $1 \mathrm{~h}$ (1:500, Thermo Fisher Scientific). Sections were mounted onto gelatincoated slides, air-dried, and coverslipped with Fluoromount G mounting medium (Electron Microscopy Sciences).

In one series of brain sections from lactating dams $(n=3)$, a tyramide signal amplification (TSA) procedure was used. Briefly, 
sections were incubated for $10 \mathrm{~min}$ in $10 \% \mathrm{H}_{2} \mathrm{O}_{2}$ to block endogenous peroxidase activity, followed by a $1 \mathrm{~h}$ blocking step in 3\% NDS. They were incubated overnight in rabbit anti-MCH $(1: 30,000)$. The next day, sections were incubated for $1 \mathrm{~h}$ in donkey anti-rabbit biotinylated secondary antibody (1:500, Jackson ImmunoResearch Laboratories AB_2340593) followed by $1 \mathrm{~h}$ in avidin-biotin complex (ABC) solution (1:500, Vector Laboratories). Finally, they were incubated in tyramide reagent $\left(1: 250\right.$, Perkin Elmer) $+0.003 \% \mathrm{H}_{2} \mathrm{O}_{2}$ followed by 30 min in AlexaFluor ${ }^{\text {TM }}$ 594-conjugated streptavidin (1:1,000, Thermo Fisher Scientific) before mounting and coverslipping with FluoroMount G mounting medium.

\section{Dual Label in situ Hybridization (ISH) and Immunohistochemistry (IHC)}

Dual-label ISH and IHC were performed to determine the colocalization of Pmch mRNA and Vgat-Cre or Vglut2-Cre GFPir (Garcia-Galiano et al., 2017). Briefly, free-floating sections from lactating females ( $n=3$ /genotype) were treated with $0.1 \%$ sodium borohydride for $15 \mathrm{~min}$ and $10 \mathrm{~min}$ with $0.25 \%$ acetic anhydride in DEPC-treated 0.1 M triethanolamine (TEA, $\mathrm{pH}$ 8.0). Sections were incubated overnight at $50^{\circ} \mathrm{C}$ in the hybridization solution containing the ${ }^{35} \mathrm{~S}-\mathrm{Pmch}$ riboprobe. Subsequently, sections were treated with RNase A for $30 \mathrm{~min}$ and submitted to stringency washes in sodium chloride-sodium citrate buffer (SSC). Sections were blocked ( $3 \%$ BSA in PBS-Triton) then incubated with antiGFP antibody $(1: 5,000)$ overnight at $4^{\circ} \mathrm{C}$. Sections were incubated for $1.5 \mathrm{~h}$ in a goat anti-chicken AlexaFluor 488 antibody and mounted onto SuperFrost plus slides. After overnight air drying, slides were dehydrated in increasing concentrations of ethanol and dipped in NTB-2 autoradiographic emulsion (Kodak/Carestream) to reflect the company merge/transition, dried and stored in light-protected boxes at $4^{\circ} \mathrm{C}$ for 3 weeks. Finally, slides were developed with D-19 developer (Kodak), dehydrated in graded ethanol, cleared in xylene, and coverslipped with DPX mounting medium.

\section{Fluorescent in situ Hybridization}

Fluorescent ISH for Pmch and Slc32a1 was performed using the RNAscope $^{\circledR}$ fluorescent multiplex detection Kit (Advanced Cell Diagnostics, cat. no. 320850) as previously described (Wang et al., 2012). Briefly, mice were deeply anesthetized with isoflurane and decapitated. Brains were collected and immediately frozen on dry ice, then cut into six series of $16-\mu \mathrm{m}$ sections on a cryostat at $-20^{\circ} \mathrm{C}$ and mounted onto Superfrost Plus slides. Slides were heated at $60^{\circ} \mathrm{C}$, then immersed in $10 \%$ buffered formalin for $2 \mathrm{~h}$ at $4^{\circ} \mathrm{C}$. They were dehydrated in increasing concentrations of ethanol, cleared in xylenes, and rehydrated in decreasing concentrations of ethanol. Next, slides were boiled for $10 \mathrm{~min}$ in sodium citrate buffer and incubated for $10 \mathrm{~min}$ in $0.03 \%$ sodium dodecyl sulfate. Slides were subsequently dried at room temperature and a hydrophobic barrier was drawn around the sections. From this point forward, steps were carried out in humidity control trays to prevent sections from drying. Endogenous peroxidase activity was blocked with a 10-min incubation with $\mathrm{H}_{2} \mathrm{O}_{2}$ followed by rinsing with nuclease-free water. RNAscope protease III was then applied and slides were heated for $30 \mathrm{~min}$ at $40^{\circ} \mathrm{C}$ before once again rinsing with nuclease-free water. Hybridization was performed by applying pre-warmed target positive and negative control probes to sections and heating for $2 \mathrm{~h}$ at $40^{\circ} \mathrm{C}$. Then, the amplification of each probe (AMP 1-2) was performed sequentially, incubating for $30 \mathrm{~min}$ at $40^{\circ} \mathrm{C}$ then rinsing in wash buffer after each Multiplex FL v2 Amp solution. The slides were developed using two RNAscope Multiplex FL v2 HRP-C $n$ solutions where $n=1-2$ (one probe per channel). For each channel, this was performed by incubating in RNAscope Multiplex FL v2 HRP-Cn for $15 \mathrm{~min}$ at $40^{\circ} \mathrm{C}$, rinsing with wash buffer, incubating for $30 \mathrm{~min}$ at $40^{\circ} \mathrm{C}$ with TSA + Fluorescein (Akoya Biosciences, cat. no. SKU NEL741001KT), incubating in RNAscope Multiplex FL v2 HRP blocker for $15 \mathrm{~min}$ at $40^{\circ} \mathrm{C}$, then rinsing once more with wash buffer. Finally, slides were counterstained with DAPI and coverslipped with ProLong Gold antifade mounting medium (ThermoFisher Scientific).

\section{Quantitative Analysis of Colocalization}

Slides from each cohort of Pmch-Cre/GFP mice were examined under an Axio Imager M2 Microscope or a SteREO DiscoveryV8 (Zeiss). The digital Allen Mouse Brain Atlas was used as a reference to determine relative location within the hypothalamus and identify the primary sites containing $\mathrm{MCH}$ immunoreactivity, GFP-immunoreactivity, and Pmch mRNA expression. Images were acquired with a digital camera (Axiocam, Zeiss) using Zen software. All sections were examined and single- and dual-labeled cells were quantified in $20 \times$ magnification using ImageJ with the Cell Counter plugin in one representative section of each area of interest (i.e., IHy, PFx, and LHA). For data illustration, only sharpness, contrast, and brightness were adjusted.

\section{RESULTS}

\section{Pmch Expression and $\mathrm{MCH}$ Immunoreactivity in Tuberal and Posterior Hypothalamus Are Similar in Naïve Male, Female, and Lactating Mice}

In situ hybridization for Pmch was performed in brain sections from sexually naïve males and females in diestrus to visualize the distribution of Pmch mRNA and assess whether it is sexually dimorphic and/or distinct in lactating females. Intense hybridization signal was observed in males in cells of the IHy, LHA, and PFx with virtually no signal elsewhere in the brain. Populations of $\mathrm{MCH}$ cells were identified in the IHy as characterized by their relatively dorsal location within the hypothalamus, just below the thalamus. Populations of $\mathrm{MCH}$ cells were defined in the LHA and PFx using other nearby structures including the fornix, optic tract, and internal capsule. There was no difference in the distribution pattern of Pmch mRNA between the two groups that could be visually ascertained (Figures 1A,B,D,E). Furthermore, the distribution of Pmch 


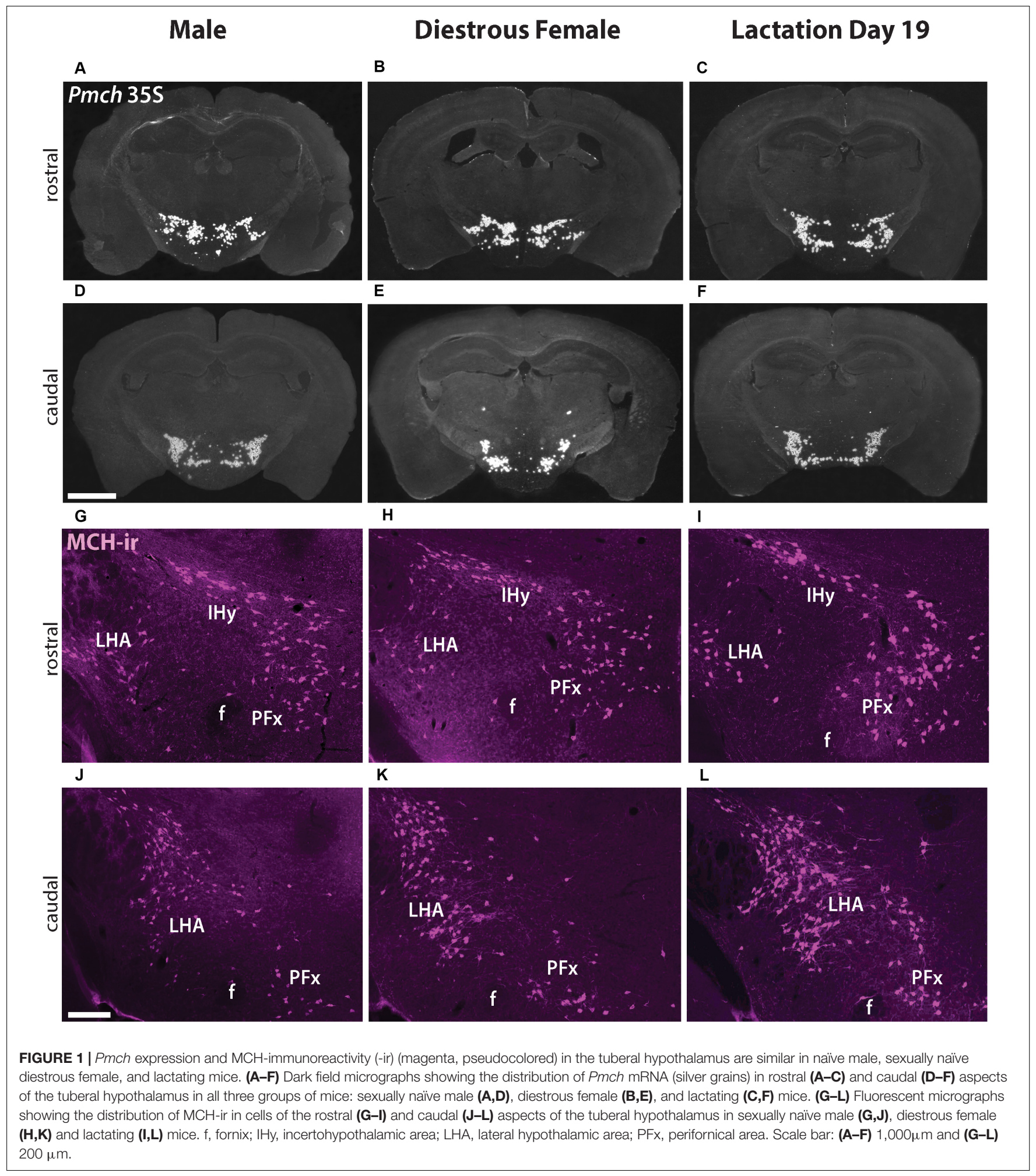

mRNA in IHy, LHA, and PFx of the lactating dams was also similar to that of the sexually naïve male and female mouse (Figures 1C,F).

To assess if the distribution of the $\mathrm{MCH}$ peptide is similar in all three groups, $\mathrm{MCH}$-immunoreactivity ( $\mathrm{MCH}$-ir) was examined and compared in sexually naïve male and female as well as lactating female mouse brains. Abundant $\mathrm{MCH}$-ir was observed in perikarya and fibers in the IHy, LHA, and PFx, in all groups in accordance with the mRNA pattern and previous studies (Bittencourt et al., 1992; Diniz et al., 2019). No clear difference 
in the distribution pattern of $\mathrm{MCH}$-ir was observed between groups (Figures 1G-L).

\section{A Subset of GFP+ Neurons in the Perifornical Area (PFx) Does Not Express MCH-ir}

Melanin-concentrating hormone- and Cre-induced GFP-ir were compared in sexually naïve male and female as well as lactating Pmch-Cre;R26-eGFP mouse brains. Virtually all GFP+ neurons expressed $\mathrm{MCH}$-ir in the IHy and LHA (Figure 2). However, a population of GFP+ neurons that do not express $\mathrm{MCH}-i$ r was consistently observed in the PFx of all groups of mice. To assess whether this was an artifact of our reporter gene, we repeated this experiment with a different reporter line. In PmchCre;eGFP-L10a mice, the same population of GFP+/MCH-cells was observed in all three groups (Figure 3). These neurons are primarily found lateral to the fornix at the level of the tuberal division of the LHA. The GFP+/MCH- cells have a distinctive small, circular morphology and cluster together near the fornix. A few such neurons were occasionally observed in other nuclei of the hypothalamus, primarily in the dorsal IHy, as well as more rostral regions such as the medial septum and prefrontal cortex, but this expression is inconsistent.

$\mathrm{MCH}$ is just one of several peptide products of the Pmch transcript, the other major one being NEI, so we evaluated NEI- and GFP-ir to determine whether the PFx GFP+/MCHneuronal population expresses NEI. Abundant NEI-ir was observed in virtually all GFP-ir perikarya and fibers in the IHy, and LHA of all three groups, showing that, as reported in rats (Bittencourt et al., 1992), virtually all hypothalamic $\mathrm{MCH}$ neurons also contain NEI. However, a population of small

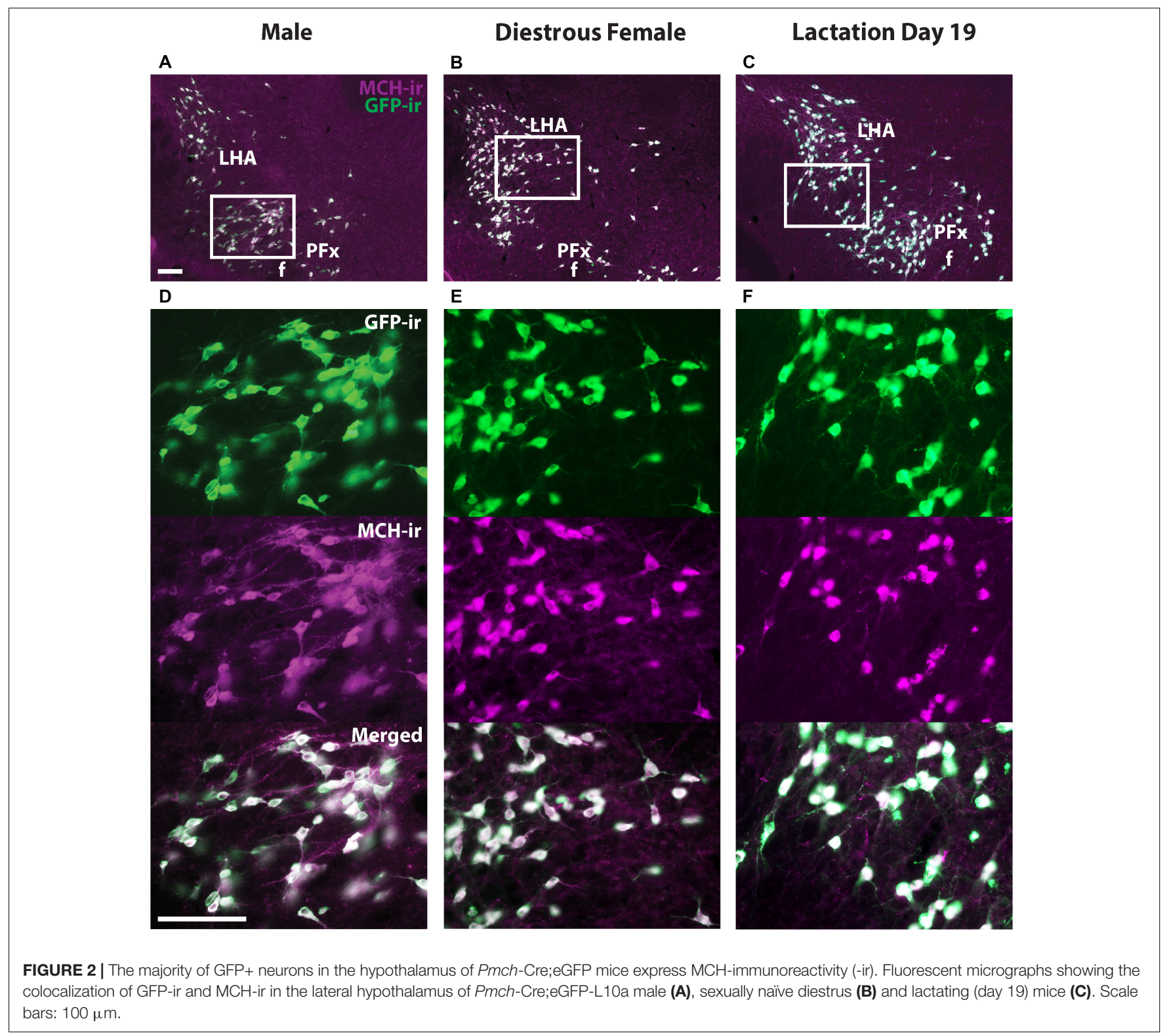




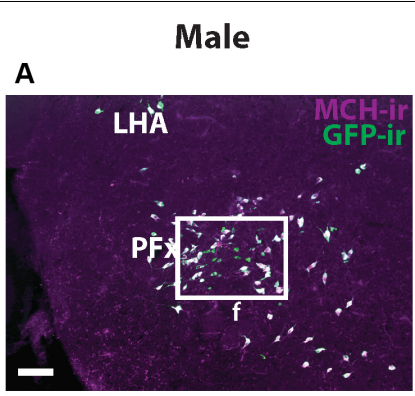

D

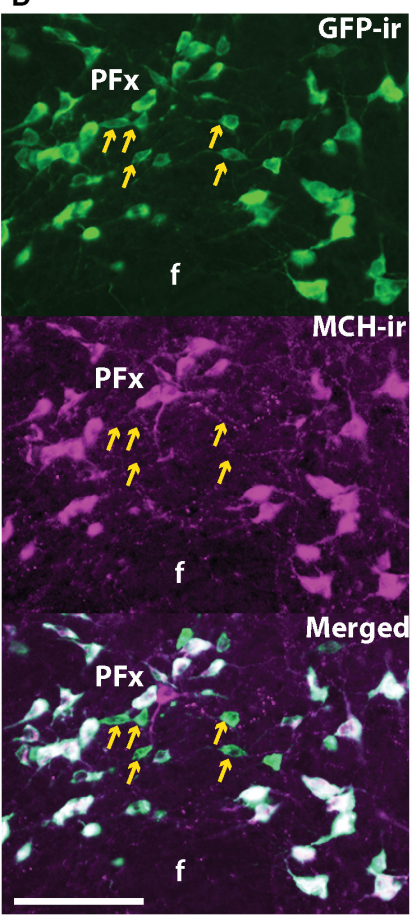

B

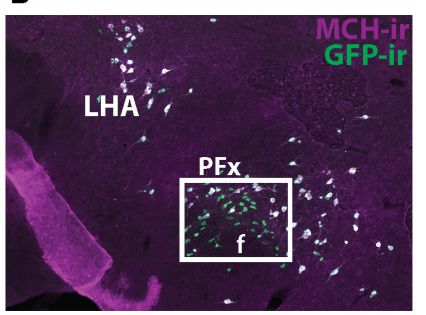

E

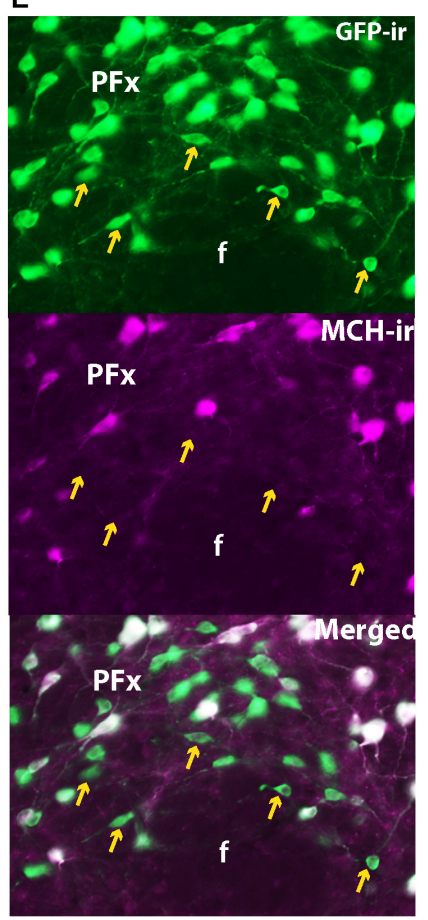

Lactation Day 19

C

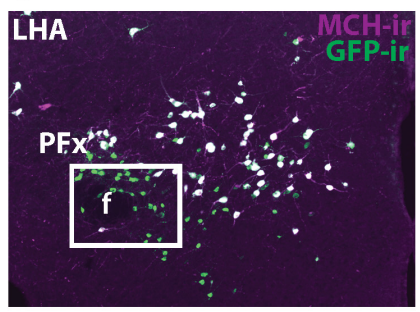

$\mathbf{F}$

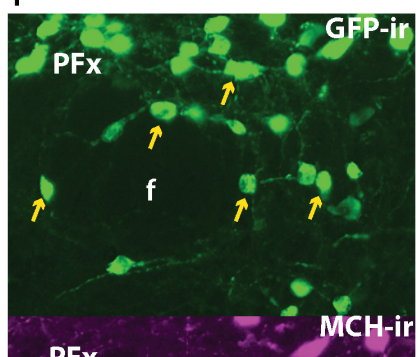

PFx

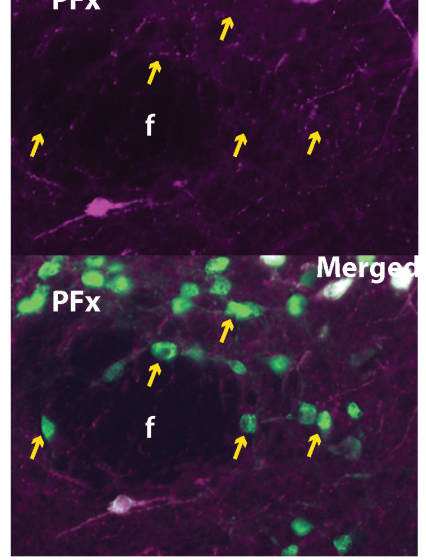

FIGURE 3 | A subset of GFP+ neurons in the perifornical area (PFx) does not express $\mathrm{MCH}$-ir. Fluorescent micrographs showing GFP-ir and $\mathrm{MCH}$-ir in the perifornical area (PFx) of a Pmch-Cre;eGFP male (A), diestrus female (B), and lactating dam (C). Note the presence of a subset of GFP-ir neurons in all groups that do not express MCH-ir (arrows). Sections are between 74 and 77 of 132 (Allen Mouse Brain Atlas, 2004). f, fornix LHA, lateral hypothalamic area; PFx, perifornical area. Scale bars: $100 \mu \mathrm{m}$.

GFP immunoreactive neurons in the PFx was also negative for NEI-ir (Figure 4). This GFP+/NEI- subset of neurons presumably overlaps with the population of $\mathrm{GFP}+/ \mathrm{MCH}-$ neurons previously described (Figure 3).

\section{Expression of Pmch and Cre-Induced GFP-ir Are Dissociated in Several Nuclei of the Rostral Forebrain in Lactating Dams}

To further map Cre-induced GFP distribution, we initially performed a comprehensive analysis of Pmch expression in the rostral forebrain of sexually naïve females in diestrus and lactating (day 19) dams. As previously described in lactating rats, we observe Pmch expression in the MPO and PVH of lactating mice. However, the pattern of mRNA distribution was distinct comparing both murine species. At the level of the anterior commissure, Pmch expression is observed in the MPO of lactating but not sexually naïve mice and is restricted to the periventricular nucleus and medial subdivision of medial preoptic nucleus (MPNm) with some lateral spreading below and above to include the anterodorsal preoptic nucleus (ADP) and the principal subdivision of the bed nucleus of the stria terminalis (BSTpr) (Figures 5A,B). Pmch mRNA was also observed in the rostral PVH of lactating but not sexually naïve mice (Figures 5D,E) and inconsistently in forebrain regions where it has not been reported in the rat. We found a moderate to dense population of Pmch cells in the lateral septum (LS) of lactating but not sexually naïve mice (Figures 5G,H) and the anterodorsal nucleus of the thalamus (AD) just lateral to the stria medullaris (Figures 5J,K).

GFP-ir was examined in rostral forebrain of lactating PmchCre;R26-eGFP and Pmch-Cre;eGFP-L10a dams to ascertain whether Pmch mRNA expression induces Cre expression in these regions. The latter reporter strain was preferred because the 


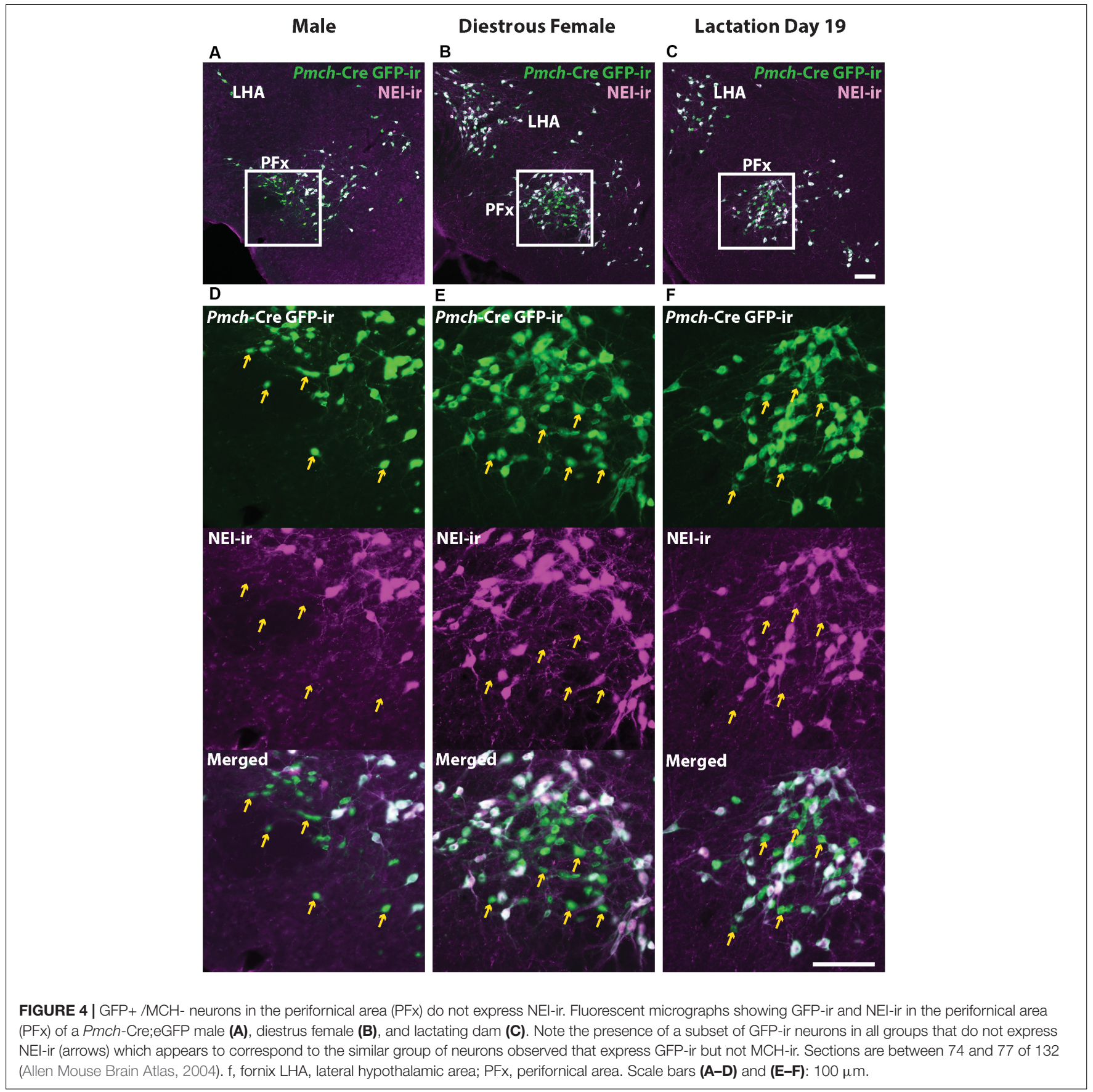

GFP fluorescence was more intense. Despite this more intense signal, little to no GFP-ir was observed in the MPO (Figure 5C) and inconsistent GFP-ir was observed in the PVH, with some animals showing strong expression while others had almost none (Figure 5F). This contrasts with the gene expression data, which shows high Pmch mRNA expression in these regions. Clear GFPir was detected in the LS (Figure 5I), and AD (Figure 5L). To assess if a delay in Cre and GFP expression was the cause of this inconsistency, a group of females was perfused 5 days after weaning (or 7 days post lactation day 19). No differences in eGFPir was observed compared to females perfused at lactation day
19 (Figure 6). The additional time for recombination and GFP expression did not yield higher GFP-ir. Collectively, our findings indicate that Pmch and Cre expression are incongruent: a large number of Pmch neurons do not express GFP-ir and a group of $\mathrm{GFP}+$ neurons in the dorsal MS does not express Pmch.

\section{Expression of VGLUT2 and VGAT Differs in Subsets of Pmch Neurons}

To gain insights into the fast neurotransmitter phenotype of $\mathrm{MCH}$ neurons we used Vgat- and Vglut2-Cre;eGFP-L10a 

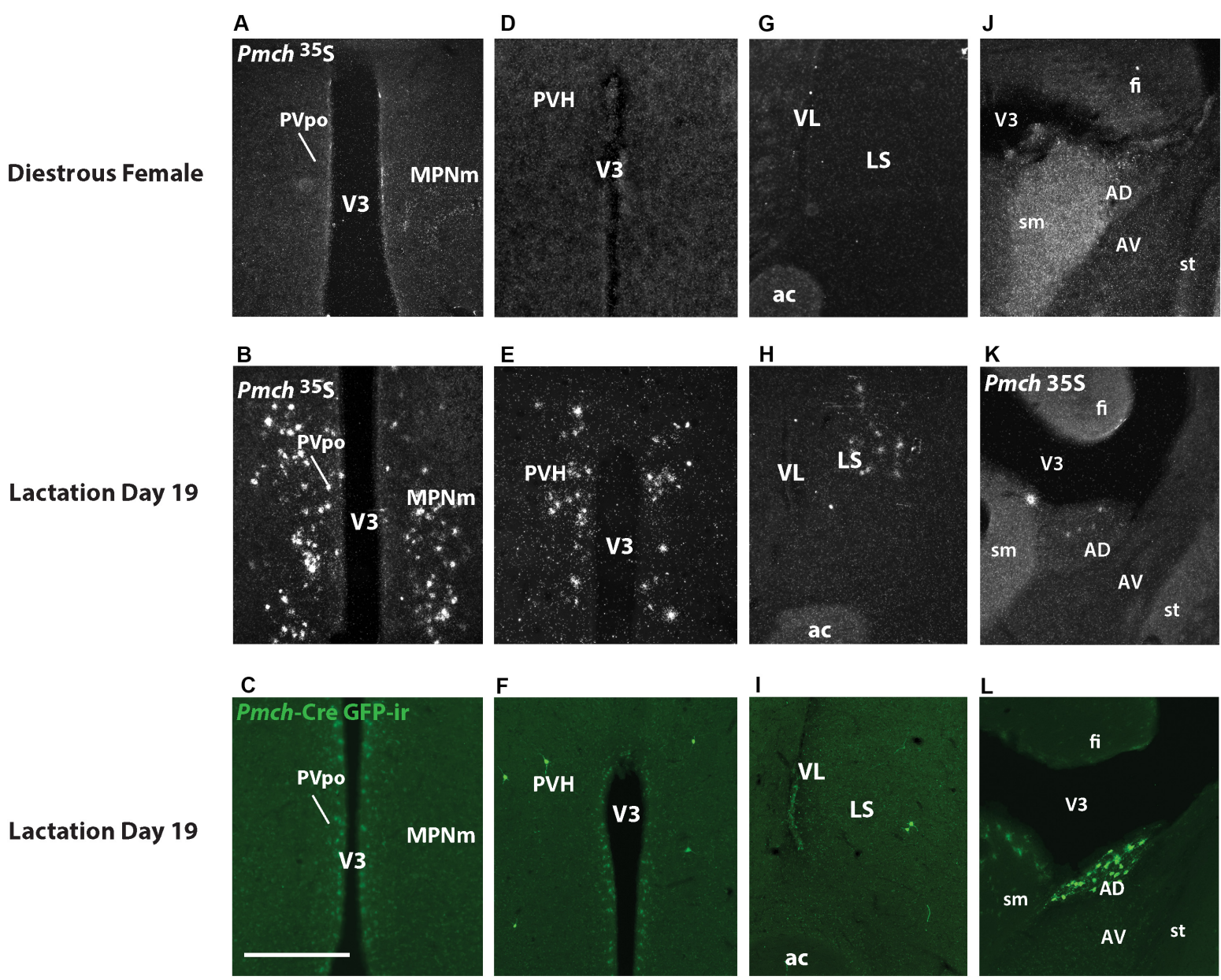

FIGURE 5 | Expression of Pmch and Cre-induced GFP-ir are dissociated in several nuclei of the rostral forebrain in lactating dams. (A) Dark field photomicrograph of the medial preoptic nucleus (MPN) of a sexually naïve female in diestrus. (B) Dark field photomicrograph of the MPN of a lactation day 19 female. (C) Fluorescent photomicrograph of the MPN of a lactation day 19 female. (D) Dark field photomicrograph of the paraventricular nucleus (PVH) of a sexually naïve female in diestrus. (E) Dark field photomicrograph of the PVH of a lactation day 19 female. (F) Fluorescent photomicrograph of the PVH of a lactation day 19 female. (G) Dark field photomicrograph of the lateral septum (LS) of a sexually naïve female in diestrus. (H) Dark field photomicrograph of the LS of a lactation day 19 female. (I) Fluorescent photomicrograph of the LS of a lactation day 19 female. (J) Dark field photomicrograph of the anterodorsal thalamic nucleus (AD) of a sexually naive female in diestrus. (K) Dark field photomicrograph of the AD of a lactation day 19 female. (L) Fluorescent photomicrograph of the AD of a lactation day 19 female. Images are taken from levels 54/132 (MPNm), 58/132 (PVH), 50/132 (LS), and 58/132 (AD) (Allen Mouse Brain Atlas, 2004). ac, anterior commissure; AV, anteroventral nucleus of the thalamus; fi, fimbria; sm, stria medullaris; st, stria terminalis; V3, third ventricle; VL, lateral ventricle. Scale bar (A-L): $200 \mu \mathrm{m}$.

reporter mice, dual label ISH and immunohistochemistry and dual label ISH.

Dual immunofluorescence was performed in brains from Vgat- and Vglut2-Cre;eGFP-L10a reporter mice to assess the colocalization of MCH-ir and GFP-ir in sexually naïve males and females in diestrus, and in nursing dams on day 19 of lactation. In the LHA, IHy, and PFx, as previously suggested by singlecell RNA sequencing data (Mickelsen et al., 2019), MCH-ir in all groups colocalized with Vglut2-Cre;eGFP (Figures 7A,C). Very few or virtually no $\mathrm{MCH}$ immunoreactive cells colocalized with Vgat-Cre;eGFP (Figures 7B,D).

In the rostral forebrain of lactating dams, MCH-ir and NEIir were low and the expression observed was highly inconsistent between animals. The Pmch-Cre induced GFP-ir that was observed did not label Pmch-expressing neurons. To evaluate co-expression with the Pmch transcript, we used brain sections from lactating Vgat- and Vglut2-Cre;eGFP reporter mice. ISH for Pmch in combination with immunohistochemical amplification of GFP fluorescent signal was performed in all three mouse groups. In the LHA, IHy, and PFx, Pmch mRNA in all groups of mice colocalized with Vglut2-Cre;eGFP primarily, and sporadically with Vgat-Cre;eGFP, consistent with our immunohistochemical data. However, in the lactating dams, colocalization was less clear. Pmch mRNA in the MPO, PVH, LS, and AD colocalized primarily with VgatCre;eGFP and not Vglut2-eGFP (Figures 8A-C). Because this result contradicts recent studies (Teixeira et al., 2019), we performed fluorescent ISH using RNAscope to further examine colocalization of Pmch with Slc32al (VGAT). The findings were unambiguous and demonstrated that virtually all Pmch 

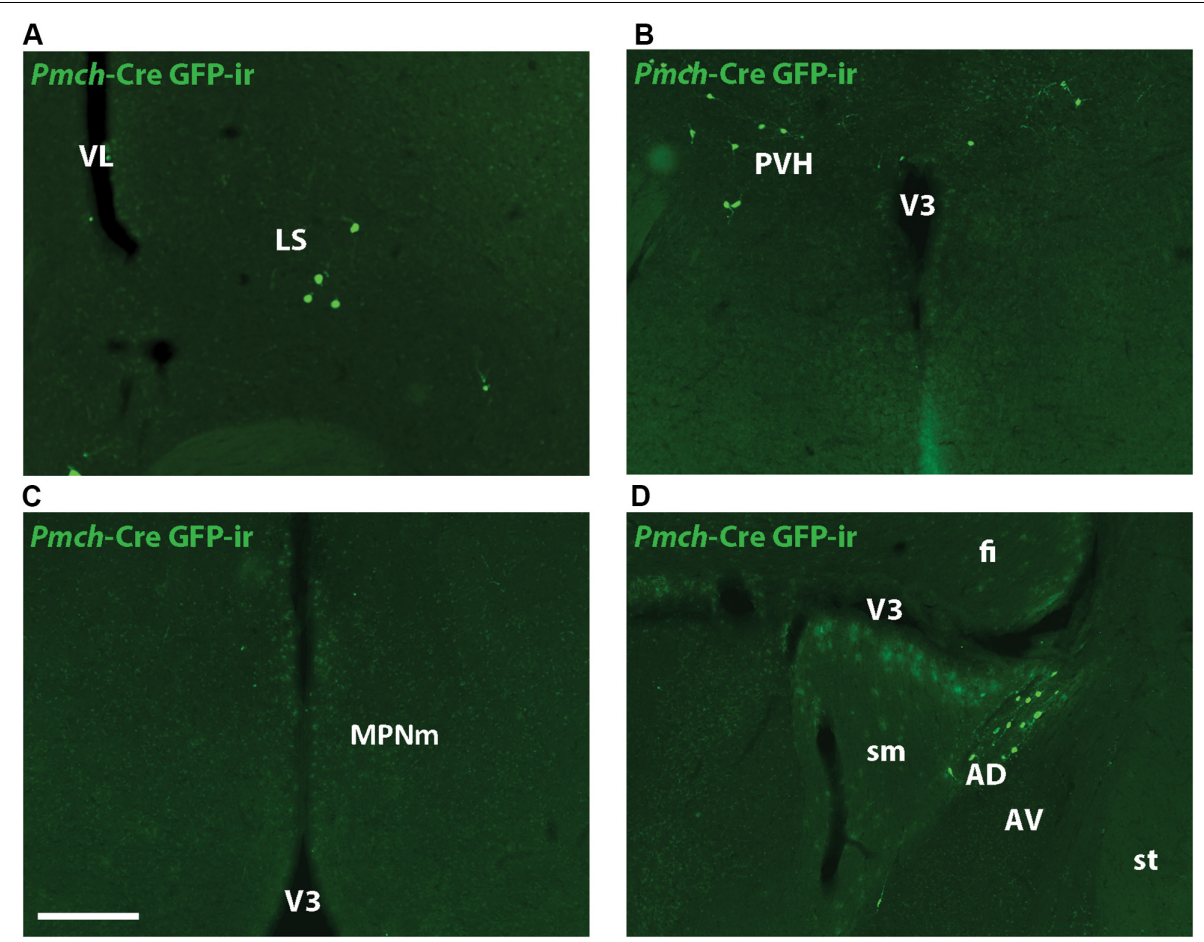

FIGURE 6 | Cre-induced GFP expression in lactation-specific regions of the rostral forebrain is unchanged post-lactation. Fluorescent micrographs showing GFP-ir in the LS (A), PVH (B), MPNm (C), and AD (D) of female mice five days post-weaning. Images are taken from levels 54/132 (MPNm), 50/132 (LS), and 58/132 (PVH/AD) (Allen Mouse Brain Atlas). AD, anterodorsal nucleus of the thalamus; AV, anteroventral nucleus of the thalamus; fi, fimbria; LS, lateral septum; MPNm, medial preoptic nucleus, medial part; sm, stria medullaris; st, stria terminalis; V3, third ventricle; VL, lateral ventricle. Scale bar (A-D): 200 $\mu \mathrm{m}$.

expressing neurons co-express Slc32a1 (Figures 8D-F). In summary, our findings indicate that $\mathrm{MCH}$ neurons in the LHA, PFx, and IHy are overwhelmingly glutamatergic, and those in the rostral forebrain induced during lactation are mostly GABAergic.

\section{DISCUSSION}

In this study, we assessed if the commercially available $\mathrm{Tg}(P m c h$ cre) 1 Lowl/J transgenic mouse line (Kong et al., 2010) is a viable model to interrogate the role of the $\mathrm{MCH}$ system in the female reproductive function. We found that in male and female (diestrous and lactating) mice, a group of Cre-induced GFP-expressing cells that do not express $\mathrm{MCH}$ - or NEI-ir is consistently observed in the PFx. In lactating dams, Pmch expression is observed in the MPO and anterior PVH, and in previously unreported forebrain nuclei, i.e., LS and AD. However, most of these sites do not show Cre-induced GFP-ir in the $\mathrm{Tg}($ Pmch-cre $) 1$ Lowl/J transgenic mouse line. We also found that typical MCH neurons in the tuberal and posterior hypothalamus co-express VGLUT2 (Slc17a6) whereas lactation-induced Pmch expression in rostral forebrain is mostly observed in VGAT (Slc32a1) neurons.

This $\operatorname{Tg}($ Pmch-cre $) 1$ Lowl/J mouse model shares many similarities with the $\mathrm{C} 57 \mathrm{BL} / 6-\mathrm{Tg}($ Pmch-cre $) 1 \mathrm{Rck} / \mathrm{J}$ line. Both lines utilize a BAC transgene. The promoter for the
C57BL/6-Tg(Pmch-cre)1Rck/J line is somewhat larger, including $108 \mathrm{~kb}$ upstream of the $\mathrm{MCH}$ gene in the BAC regulatory element as opposed to the $64 \mathrm{~kb}$ in the $\mathrm{Tg}($ Pmch-cre $) 1 \mathrm{Lowl} / \mathrm{J}$. The strains also have nearly identical penetrance and specificity (Kong et al., 2010; Jego et al., 2013). Ultimately, we chose to focus on the $\mathrm{Tg}($ Pmch-cre)1Lowl/J line because it is used and cited more frequently, making our findings applicable to more researchers in the field (Kong et al., 2010; Varin et al., 2018; Dilsiz et al., 2020).

Whereas most previous studies have been focused on peptide distribution, here we gave special attention to the distribution and expression of the Pmch gene. As previously described for $\mathrm{MCH}$-ir, Pmch expression is very similar in naïve male and female mice with clear anterior and posterior patterns of distribution (Diniz et al., 2019). The anterior pattern of mRNA expression is characterized by distinct populations of neurons in the IHy, PFx, and a few neurons in the LHA (anterior division), while the posterior pattern primarily consists of dense expression in the LHA (tuberal division) and PFx. We have further shown that virtually all $\mathrm{MCH}$-ir cells express GFP (and thus Cre recombinase) in the Pmch-Cre reporter mouse, indicating that Cre expression in the $\mathrm{Tg}($ Pmch-Cre $) 1$ Lowl/J BAC transgenic mouse line is highly penetrant. It is worth mentioning that we observe higher penetrance than what was demonstrated in the originating publication (Kong et al., 2010). This is likely due to the use of a ribosomal protein-associated reporter line, which is more concentrated in the soma, whereas the original publication validated the model used a TdTomato reporter, which 


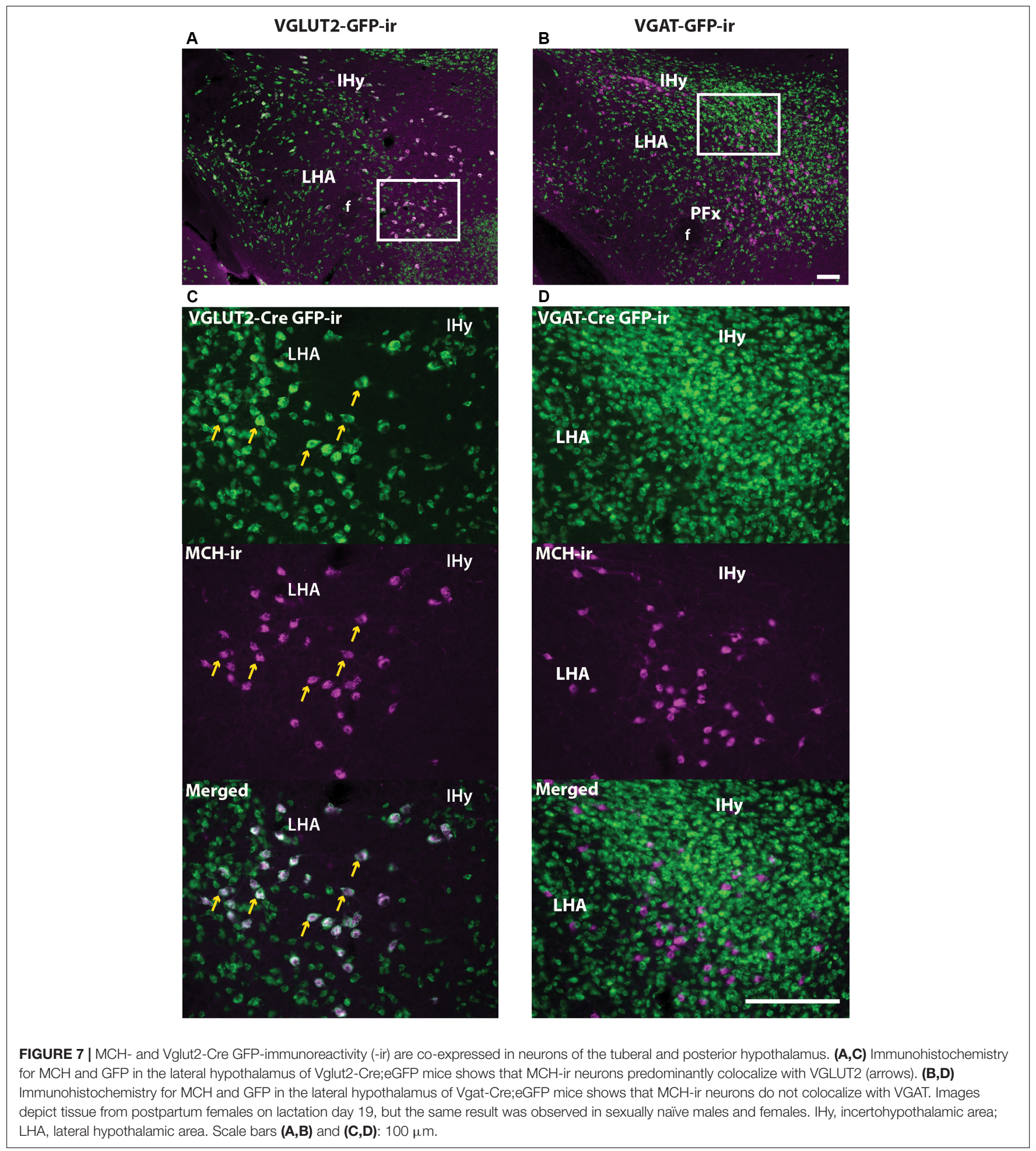

is expressed in fibers and thus yields a more diluted signal (Madisen et al., 2010; Liu et al., 2014).

However, we observe some GFP-ir cells which do not express $\mathrm{MCH}$ located in the PFx. The cause for this discrepancy is not evident, but an artifact (ectopic expression) of the BAC transgene is a reasonable explanation, calling into question the specificity of Cre expression. Alternatively, $\mathrm{MCH}$ could play a developmental role in these cells. If its production is turned off by adulthood, MCH-ir and Pmch would not be detected by our assays, yet GFP signal would still be visible as it is driven by a ubiquitous promoter. Future work will be necessary to address this question by characterizing hypothalamic Pmch 


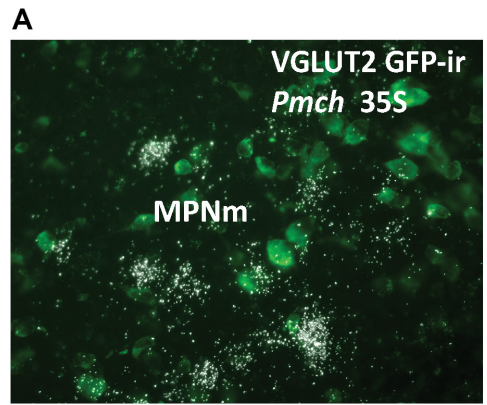

D

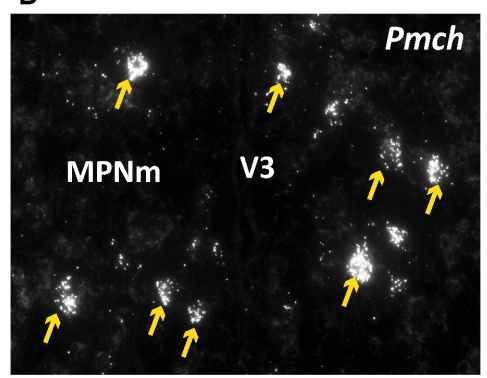

\section{B}

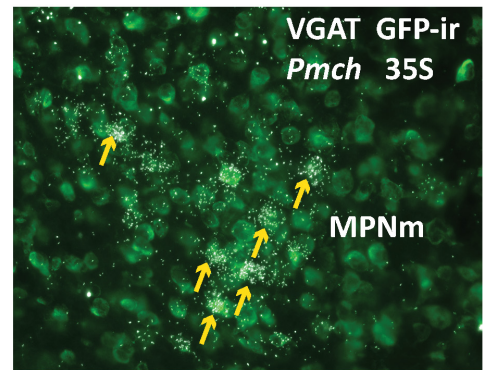

E

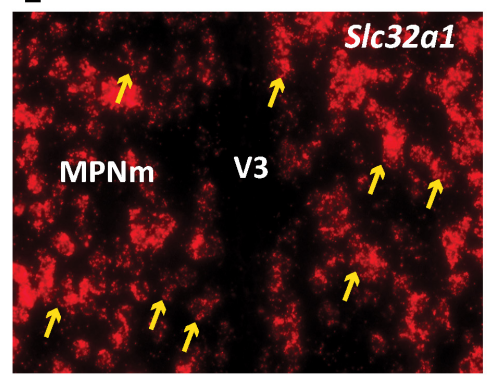

C

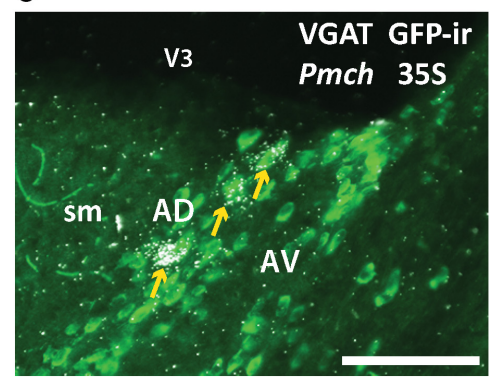

$\mathbf{F}$

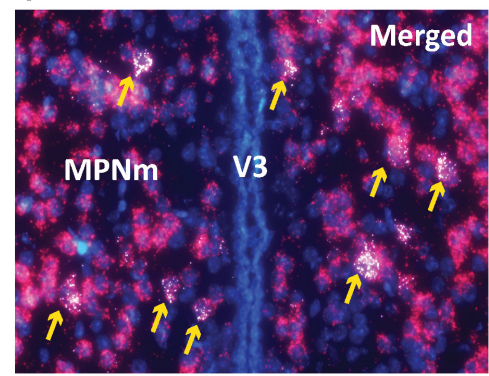

FIGURE 8| Pmch is expressed in rostral forebrain neurons that co-express Slc32a1 (VGAT). (A,B) Fluorescent and dark field micrographs showing the majority of Pmch in the MPN do not colocalize with VGLUT2 (A) but do colocalize with VGAT (B). (C) Fluorescent and dark field micrograph showing the majority of Pmch in the AD also colocalizes with VGAT. (D-F) Fluorescent micrographs showing co-expression (arrows) of Pmch (white) and S/c32a1 (red) in medial aspect of the medial preoptic nucleus (MPNm) using RNAscope ${ }^{\circledR}$ technology. DAPI counterstain in blue. AD, anterodorsal nucleus of the thalamus; AV, anteroventral nucleus of the thalamus; MPNm, medial preoptic nucleus, medial part; sm, stria medullaris; VL, lateral ventricle; V3, third ventricle. Scale bars (A-F): $100 \mu$ m.

mRNA and MCH peptide expression at various timepoints in the developing mouse brain.

In lactating mice, Pmch expression is clearly different from rats (Knollema et al., 1992). While in rats, Pmch expression is triggered at mid-lactation and becomes dense in the MPN and $\mathrm{PVH}$, in mice, Pmch is observed only at the end of lactation (day 19) at low to moderate levels specifically in the MPNm (Teixeira et al., 2019) and anterior PVH. Notably, the mice also show lactation-induced Pmch in previously unreported brain sites, including the LS, the ADP, the ventral BSTpr and the anterior $\mathrm{AD}$. The role of $\mathrm{MCH}$ system in these sites is not known. Furthermore, the inconsistency of Cre-mediated GFP expression indicate that the Pmch-Cre BAC transgenic mouse model is not useful for investigating transient $\mathrm{MCH}$ expression in lactating dams. Euthanizing lactating dams 5 days after weaning the offspring to allow more time for Cre-mediated recombination and GFP expression did not change the low to non-existent levels of GFP-ir in the MPNm, PVH and LS of the lactating dam. Researchers should also note that dams euthanized on lactating day 19 or 7 days post-weaning exhibit ectopic GFP expression in brain sites we did not observe Pmch mRNA. Use of female breeders in studies of $\mathrm{MCH} / \mathrm{NEI}$ function may generate unreliable data.

Turning on $\mathrm{MCH}$ production for just several days at the end of lactation suggests that its role is highly specific and timesensitive. If this is the case, it may indicate that $\mathrm{MCH}$ in these neurons is subjected to unique epigenetic regulation that may not be reproduced in the BAC sequence. Moreover, differences at the level of transcription, such as the use of enhancers, which inform $\mathrm{MCH}$ production and processing could interfere with Cre expression in these cells if the enhancers are not located within the BAC transgene. A BAC genomic clone containing $64 \mathrm{~kb}$ upstream and $34 \mathrm{~kb}$ downstream of Pmch gene was used to generate the original $\mathrm{MCH}$-Cre mice with the goal of capturing most of the gene's regulatory elements (Kong et al., 2010). However, mammalian enhancers can be located as far as one million base pairs from the transcriptional start site of the gene in question (Dean, 2006; Maston et al., 2006; Krivega and Dean, 2012). Thus, it is possible that important enhancer activity has been excluded from the BAC regulatory element.

It has been suggested that prolactin-induced phosphorylation of STAT5 (pSTAT5) has a role in the lactation-induced MCH-ir in the MPO. STAT5 is a known activator protein for hundreds of enhancers, particularly those implicated in pregnancy- and lactation-associated genes (Yamaji et al., 2013; Teixeira et al., 2019). The seven STAT family proteins are all believed to target the same palindromic core motif, TTCN ${ }_{2-4}$ GAA. STAT5A and $B$ in particular have a strong preference for palindromic core motifs of $\mathrm{N}_{3}$ (Ehret et al., 2001). A brief search using DNASTAR Lasergene software shows that approximately 50 sites per $100 \mathrm{~kb}$ contain sequence $\mathrm{TTCN}_{3} \mathrm{GAA}$, yielding close to 500 potential pSTAT5 binding sites upstream of the Pmch gene regulatory element that are potentially missing from the BAC regulatory elements. Whereas these are clearly speculations, it is possible that regulatory sequences essential for Pmch expression during lactation are not included in the BAC used in this mouse model.

For the purposes of anatomical and specific functional studies, i.e., viral injections, tract tracing or colocalization with other 
transcripts and peptides, this may be an acceptable model because nearly all of the Cre-expressing cells are indeed $\mathrm{MCH}$ positive neurons with the exception of the small, highly localized population of PFx neurons that are easy to identify.

It has long been debated whether $\mathrm{MCH}$ neurons release GABA, glutamate, both, or neither of the classical fast neurotransmitters. In rats, it has been documented that $\mathrm{MCH}$ neurons of the tuberal hypothalamus express Gad1 (glutamic acid decarboxylase or GAD67), the enzyme that catalyzes the decarboxylation of glutamate to GABA, and the vesicular GABA transporter (VGAT) can be found in $\mathrm{MCH}$ terminals (Sapin et al., 2010; del Cid-Pellitero and Jones, 2012). However, glutamate has been observed in $\mathrm{MCH}$ cells and demonstrated to be released from MCH terminals (Chee et al., 2015; Schneeberger et al., 2018). Using a different reporter system, however, others claim that these neurons are neither GABAergic nor glutamatergic (Blanco-Centurion et al., 2019). The glutamatergic-leaning transcriptional profile of hypothalamic MCH neurons has largely been reinforced with the availability of advanced genomic techniques, i.e., single-cell RNA sequencing (Mickelsen et al., 2019). Our data using distinct methodologies is in agreement with the latter and demonstrates that the typical $\mathrm{MCH}$ neurons in the tuberal hypothalamus are essentially glutamatergic. It is also worth mentioning that our findings are not contradictory to previous studies in rats showing that $\mathrm{MCH}$ neurons coexpress GAD67. Species differences should not be ignored as the colocalization of $\mathrm{MCH}$ and GAD67 or lack thereof in mice has not been shown. The presence of GAD67 indicates that the cell has the necessary machinery to synthetize GABA from glutamate (Roberts and Frankel, 1950; Martin, 1987). Whether this process is observed in specific physiological state(s) in $\mathrm{MCH}$ neurons of the mouse hypothalamus needs further investigation.

Transient MCH expression in the rat MPO, on the other hand, has been shown to occur in GAD67 expressing neurons (Rondini et al., 2010). Using immunohistochemistry and radioactive ISH we show that transient lactation-induced $\mathrm{MCH}$ expression is largely restricted to VGAT + neurons, though in the MPO sporadic Pmch mRNA can apparently be also found in VGLUT2 + neurons. RNAscope confirms that most of lactation-induced Pmch neurons co-express Slc32al (VGAT). This contrast in fast neurotransmitter phenotype could underlie fundamental differences in the function and properties of these transient $\mathrm{MCH}$ neurons as compared with their counterparts elsewhere in the hypothalamus. However, it also contradicts the recent finding that $\mathrm{MCH}$ neurons in the LHA, PFx, and IHy express neither Vglut2, Vglut3, nor Vgat (Teixeira et al., 2019). As aforementioned, this difference may arise from our use of an eGFP-L10a reporter strain rather than a TdTomato reporter. Because L10 is a ribosomal protein, it concentrates in the cytoplasm for ease of visualization whereas TdTomato also labels terminals, diluting the signal (Madisen et al., 2010; Liu et al., 2014).

We conclude that the Pmch-Cre mouse model is unsuitable for studying the role of $\mathrm{MCH}$ during lactation. Any Credependent manipulations will not target the neurons which transiently express $\mathrm{MCH}$ during lactation, especially in the
MPNm, PVH and LS, where the mRNA/GFP-ir discrepancy is most pronounced. This presents a need to develop other methods for investigating these neurons, which could involve the development of another transgenic mouse line. It would be particularly beneficial to use a knock-in strategy to increase specificity and congruence of Cre and Pmch expression in both sexes and all physiological states.

\section{Resource Identification Initiative}

RRID described in methods session.

\section{Life Science Identifiers}

This study uses genetically modified mouse models purchased from JAX mice. The nomenclature is in agreement with the International Committee on Standardized Genetic Nomenclature for Mice.

\section{DATA AVAILABILITY STATEMENT}

Requests to access the datasets should be directed to cfelias@umich.edu.

\section{ETHICS STATEMENT}

The animal study was reviewed and approved by IACUC, University of Michigan.

\section{AUTHOR CONTRIBUTIONS}

$\mathrm{BB}, \mathrm{WF}$, and $\mathrm{CE}$ designed the study. BB and WF carried out the experiments. TB designed and generated the Pmch probe for radioactive in situ hybridization. DG-G assisted with probe design and execution of radioactive in situ hybridization. SA contributed with maintenance and genotyping of the Pmch-Cre mouse colony. GV contributed with maintenance and genotyping of the Vgat- and Vglut2-Cre;eGFP mouse colonies. BB analyzed data and compiled the manuscript. CE, GV, and SA contributed to manuscript revisions. All authors contributed to the article and approved the submitted version.

\section{FUNDING}

This study was funded by NIH CTRB T32 (BB), NIH R01HD 069702 (to CE), and NIH R21 HD 090567 (to CE and GV).

\section{ACKNOWLEDGMENTS}

We want to thank Dr. David P. Olson (University of Michigan) for the donation of the eGFP-L10a mouse line and Dr. Paul Sawchenko (Salk Institute) for the donation of anti-NEI antisera. We also thank Dr. Jackson Bittencourt and Dr. Jose Donato Jr. (University of São Paulo) for insightful discussions. 


\section{REFERENCES}

Allen Mouse Brain Atlas (2004). Allen Institute for Brain Science. Allen Mouse Brain Atlas, version 2 2011. Available online at: https://mouse.brain-map.org/ (accessed July 1, 2020).

Attademo, A. M., Rondini, T. A., Rodrigues, B. C., Bittencourt, J. C., Celis, M. E., and Elias, C. F. (2006). Neuropeptide glutamic acid-isoleucine may induce luteinizing hormone secretion via multiple pathways. Neuroendocrinology. 83, 313-324. doi: 10.1159/000096052

Bahjaoui-Bouhaddi, M., Fellmann, D., Griffond, B., and Bugnon, C. (1994). Insulin treatment stimulates the rat melanin-concentrating hormone-producing neurons. Neuropeptides 27, 251-258. doi: 10.1016/0143-4179(94)90006-x

Baker, B. I., Bird, D. J., and Buckingham, J. C. (1985). Salmonid melanin-concentrating hormone inhibits corticotrophin release. J. Endocrinol.106, R5-R8.

Bittencourt, J. C., and Elias, C. F. (1998). Melanin-concentrating hormone and neuropeptide EI projections from the lateral hypothalamic area and zona incerta to the medial septal nucleus and spinal cord: a study using multiple neuronal tracers. Brain Res. 805, 1-19. doi: 10.1016/s0006-8993(98) 00598-8

Bittencourt, J., Presse, F., Arias, C., Peto, C., Vaughan, J., Nahon, J. L., et al. (1992). The melanin-concentrating hormone system of the rat brain: an immuno-and hybridization histochemical characterization. J. Comp. Neurol. 319, 218-245. doi: 10.1002/cne.903190204

Blanco-Centurion, C., Luo, S., Spergel, D. J., Vidal-Ortiz, A., Oprisen, S., Van den Pol, A. N., et al. (2019). Dynamic network activation of hypothalamic mch neurons in rem sleep and exploratory behavior. J. Neurosci. 39, 0305-0319.

Casatti, C. A., Elias, C. F., Sita, L. V., Frigo, L., Furlani, V. C., Bauer, J. A., et al. (2002). Distribution of melanin-concentrating hormone neurons projecting to the medial mammillary nucleus. Neuroscience 115, 899-915. doi: 10.1016/ s0306-4522(02)00508-0

Chee, M. J., Arrigoni, E., and Maratos-Flier, E. (2015). Melanin-concentrating hormone neurons release glutamate for feedforward inhibition of the lateral septum. J. Neurosci. 35, 3644-3651. doi: 10.1523/jneurosci.4187-14.2015

Dean, A. (2006). On a chromosome far, far away: LCRs and gene expression. Trends Genet. 22, 38-45. doi: 10.1016/j.tig.2005.11.001

del Cid-Pellitero, E., and Jones, B. E. (2012). Immunohistochemical evidence for synaptic release of GABA from melanin-concentrating hormone containing varicosities in the locus coeruleus. Neuroscience 223, 269-276. doi: 10.1016/j. neuroscience.2012.07.072

Dilsiz, P., Aklan, I., Sayar Atasoy, N., Yavuz, Y., Filiz, G., Koksalar, F., et al. (2020). MCH neuron activity is sufficient for reward and reinforces feeding. Neuroendocrinology 110, 258-270. doi: 10.1159/000501234

Diniz, G., Battagello, D., Cherubini, P., Reyes-Mendoza, J., Luna-Illades, C., Klein, M., et al. (2019). Melanin-concentrating hormone peptidergic system: comparative morphology between muroid species. J. Comp. Neurol. 527, 2973 3001. doi: $10.1002 / \mathrm{cne} .24723$

Ehret, G. B., Reichenbach, P., Schindler, U., Horvath, C. M., Fritz, S., Nabholz, M., et al. (2001). DNA binding specificity of different STAT proteins. Comparison of in vitro specificity with natural target sites. J. Biol. Chem. 276, 6675-6688. doi: $10.1074 /$ jbc.m001748200

Elias, C. F., and Bittencourt, J. C. (1997). Study of the origins of melaninconcentrating hormone and neuropeptide EI immunoreactive projections to the periaqueductal gray matter. Brain Res. 755, 255-271. doi: 10.1016/s00068993(97)00104-2

Elias, C. F., Saper, C. B., Maratos-Flier, E., Tritos, N. A., Lee, C., Kelly, J., et al. (1998). Chemically defined projections linking the mediobasal hypothalamus and the lateral hypothalamic area. J. Comp. Neurol. 402, 442-459. doi: 10.1002/ (sici)1096-9861(19981228)402:4<442::aid-cne2>3.0.co;2-r

Frazão, R., Cravo, R. M., Donato, J., Ratra, D. V., Clegg, D. J., Elmquist, J. K., et al. (2013). Shift in Kiss1 cell activity requires estrogen receptor $\alpha . J$. Neurosci. 33, 2807-2820. doi: 10.1523/jneurosci.1610-12.2013

Garcia-Galiano, D., Borges, B. C., Donato, J., Allen, S. J., Bellefontaine, N., Wang, M., et al. (2017). PI3K $\alpha$ inactivation in leptin receptor cells increases leptin sensitivity but disrupts growth and reproduction. JCI Insight 12:e96728.

Jego, S.D., Glasgow, S., Gutierrez Herrera, C., Ekstrand, M., Reed, S., Boyce, R., et al (2013). Optogenetic identification of a rapid eye movement sleep modulatory circuit in the hypothalamus. Nat Neurosci. 16, 1637-1643. doi: 10.1038/nn. 3522
Knollema, S., Brown, E. R., Vale, W., and Sawchenko, P. E. (1992). Novel hypothalamic and preoptic sites of prepro-melanin-concentrating hormone messenger ribonucleic Acid and Peptide expression in lactating rats. J. Neuroendocrinol. 4, 709-717. doi: 10.1111/j.1365-2826.1992.tb00222.x

Kong, D., Vong, L., Parton, L. E., Ye, C., Tong, Q., Hu, X., et al. (2010). Glucose stimulation of hypothalamic $\mathrm{MCH}$ neurons involves $\mathrm{K}(\mathrm{ATP})$ channels, is modulated by UCP2, and regulates peripheral glucose homeostasis. Cell Metab. 12, 545-552. doi: 10.1016/j.cmet.2010.09.013

Krivega, I., and Dean, A. (2012). Enhancer and promoter interactions-long distance calls. Curr. Opin. Genet. Dev. 22, 79-85. doi: 10.1016/j.gde.2011.11.001

Liu, J., Krautzberger, A. M., Sui, S. H., Hofmann, O. M., Chen, Y., Baetscher, M., et al. (2014). Cell-specific translational profiling in acute kidney injury. J. Clin. Invest. 124, 1242-1254. doi: 10.1172/jci72126

Madisen, L., Zwingman, T. A., Sunkin, S. M., Oh, S. W., Zariwala, H. A., Gu, H., et al. (2010). A robust and high-throughput Cre reporting and characterization system for the whole mouse brain. Nat. Neurosci. 13, 133-140. doi: 10.1038/nn. 2467

Mao, X., Fujiwara, Y., Chapdelaine, A., Yang, H., and Orkin, S. H. (2001). Activation of EGFP expression by Cre-mediated excision in a new ROSA26 reporter mouse strain. Blood 97, 324-326. doi: 10.1182/blood.v97.1.324

Martin, D. L. (1987). Regulatory properties of brain glutamate decarboxylase. Cell Mol. Neurobiol. 7, 237-253. doi: 10.1007/bf00711302

Maston, G. A., Evans, S. K., and Green, M. R. (2006). Transcriptional regulatory elements in the human genome. Annu. Rev. Genomics Hum. Genet. 7, 29-59. doi: 10.1146/annurev.genom.7.080505.115623

Mickelsen, L. E., Bolisetty, M., Chimileski, B. R., Fujita, A., Beltrami, E. J., Costanzo, J. T., et al. (2019). Single-cell transcriptomic analysis of the lateral hypothalamic area reveals molecularly distinct populations of inhibitory and excitatory neurons. Nat. Neurosci. 04, 642-656. Epub 2019/03/11. doi: 10.1038/ s41593-019-0349-8

Mohsen, Z., Sim, H., Garcia-Galiano, D., Han, X., Bellefontaine, N., Saunders, T. L., et al. (2017). Sexually dimorphic distribution of Prokr2 neurons revealed by the Prokr2-Cre mouse model. Brain Struct. Funct. 222, 4111-4129. doi: 10.1007/s00429-017-1456-5

Qu, D., Ludwig, D. S., Gammeltoft, S., Piper, M., Pelleymounter, M. A., Cullen, M. J., et al. (1996). A role for melanin-concentrating hormone in the central regulation of feeding behaviour. Nature 380, 243-247. doi: 10.1038/3802 $43 \mathrm{a} 0$

Roberts, E., and Frankel, S. (1950). gamma-Aminobutyric acid in brain: its formation from glutamic acid. J. Biol. Chem. 187, 55-63.

Rondini, T. A., Donato, J., Rodrigues, B. C., Bittencourt, J. C., and Elias, C. F. (2010). Chemical identity and connections of medial preoptic area neurons expressing melanin-concentrating hormone during lactation. J. Chem. Neuroanat. 39, 51-62. doi: 10.1016/j.jchemneu.2009.10.005

Sapin, E., Bérod, A., Léger, L., Herman, P. A., Luppi, P. H., and Peyron, C. (2010). A very large number of GABAergic neurons are activated in the tuberal hypothalamus during paradoxical (REM) sleep hypersomnia. PLoS One 5:e11766. doi: 10.1371/journal.pone.0011766

Schneeberger, M., Tan, K., Nectow, A. R., Parolari, L., Caglar, C., Azevedo, E., et al. (2018). Functional analysis reveals differential effects of glutamate and MCH neuropeptide in MCH neurons. Mol. Metab. 07, 83-89. Epub 2018/05/08. doi: 10.1016/j.molmet.2018.05.001

Sibony, M., Commo, F., Callard, P., and Gasc, J. (1995). Enhancement of mRNA in situ hybridization signal by microwave heating. Lab. Invest. 73, 586-591.

Sita, L., Elias, C., and Bittencourt, J. (2007). Connectivity pattern suggests that incerto-hypothalamic area belongs to the medial hypothalamic system. Neuroscience 148, 949-969. doi: 10.1016/j.neuroscience.2007.07.010

Teixeira, P. D. S., Wasinski, F., Lima, L. B., Frazão, R., Bittencourt, J. C., and Donato, J. (2019). Regulation and neurochemical identity of melaninconcentrating hormone neurons in the preoptic area of lactating mice. J. Neuroendocrinol. 32:e12818.

Varin, C., Luppi, P. H., and Fort, P. (2018). Melanin-concentrating hormoneexpressing neurons adjust slow-wave sleep dynamics to catalyze paradoxical (REM) sleep. Sleep 41, 1-12.

Verret, L., Goutagny, R., Fort, P., Cagnon, L., Salvert, D., Leger, L., et al. (2003). A role of melanin-concentrating hormone producing neurons in the central regulation of paradoxical sleep. BMC Neurosci. 9:19. doi: 10.1186/14712202-4-19 
Vong, L., Ye, C., Yang, Z., Choi, B., Chua, S., and Lowell, B. B. (2011). Leptin action on GABAergic neurons prevents obesity and reduces inhibitory tone to POMC neurons. Neuron. 71, 142-154. doi: 10.1016/j.neuron.2011. 05.028

Wang, F., Flanagan, J., Su, N., Wang, L. C., Bui, S., Nielson, A., et al. (2012). RNAscope: a novel in situ RNA analysis platform for formalin-fixed, paraffinembedded tissues. J. Mol. Diagn. 14, 22-29.

Yamaji, D., Kang, K., Robinson, G. W., and Hennighausen, L. (2013). Sequential activation of genetic programs in mouse mammary epithelium during pregnancy depends on STAT5A/B concentration. Nucleic Acids Res. 41, 16221636. doi: $10.1093 /$ nar/gks1310
Conflict of Interest: The authors declare that the research was conducted in the absence of any commercial or financial relationships that could be construed as a potential conflict of interest.

Copyright $\odot 2020$ Beekly, Frankel, Berg, Allen, Garcia-Galiano, Vanini and Elias. This is an open-access article distributed under the terms of the Creative Commons Attribution License (CC BY). The use, distribution or reproduction in other forums is permitted, provided the original author(s) and the copyright owner(s) are credited and that the original publication in this journal is cited, in accordance with accepted academic practice. No use, distribution or reproduction is permitted which does not comply with these terms. 